\title{
Isocitrate dehydrogenase status and molecular subclasses of glioma and glioblastoma
}

\author{
Sameer Agnihotri, Ph.D., ${ }^{1}$ Kenneth D. Aldape, M.D., ${ }^{3}$ \\ and Gelareh Zadeh, M.D., PH.D. ${ }^{1,2}$ \\ ${ }^{1}$ Arthur and Sonia Labatt Brain Tumour Research Centre, The Hospital for Sick Children; ${ }^{2}$ Division of \\ Neurosurgery, Toronto Western Hospital, University of Toronto, Ontario, Canada; and ${ }^{3}$ Department of \\ Pathology, The University of Texas M.D. Anderson Cancer Center, Houston, Texas
}

\begin{abstract}
Diffuse gliomas and secondary glioblastomas (GBMs) that develop from low-grade gliomas are a common and incurable class of brain tumor. Mutations in the metabolic enzyme glioblastomas (IDH1) represent a distinguishing feature of low-grade gliomas and secondary GBMs. IDH1 mutations are one of the most common and earliest detectable genetic alterations in low-grade diffuse gliomas, and evidence supports this mutation as a driver of gliomagenesis. Here, the authors highlight the biological consequences of IDH1 mutations in gliomas, the clinical and therapeutic/diagnostic implications, and the molecular subtypes of these tumors. They also explore, in brief, the non-IDH1-mutated gliomas, including primary GBMs, and the molecular subtypes and drivers of these tumors. A fundamental understanding of the diversity of GBMs and lower-grade gliomas will ultimately allow for more effective treatments and predictors of survival.

(http://thejns.org/doi/abs/10.3171/2014.9.FOCUS14505)
\end{abstract}

KeY Words • glioblastoma • IDH1 • tumor metabolism

$\mathrm{G}$ Lioblastoma (GBM) is the most common brain tumor type, and despite surgery, radiation, and chemotherapy, it is incurable..$^{27,64}$ The majority of GBMs, $88 \%-90 \%$, arise de novo and are classified as Grade IV gliomas according to WHO criteria. ${ }^{36}$ These tumors are often present in elderly patients without any clinical evidence of a less malignant precursor tumor. Conversely, secondary GBMs progress from lowgrade gliomas (WHO Grade II-III); these tumors arise in younger patients and are often associated with a better prognosis. Although histologically indistinguishable, primary and secondary GBMs greatly differ at the genetic and epigenetic levels. One such distinguishing feature is the identification of mutations in the metabolic enzyme isocitrate dehydrogenase 1 (IDH1). IDH1 mutations are thought to be one of the earliest detectable genetic alterations in low-grade diffuse gliomas. In this review, we highlight the biological consequences of IDH1 mutations

Abbreviations used in this paper: $2 \mathrm{HG}=2$-hydroxyglutarate; $\alpha-\mathrm{KG}=\alpha$-ketoglutarate; $\mathrm{CDK}=$ cyclin-dependent kinase; CIMP $=\mathrm{CpG}$ island hypermethylator phenotype; DNMT = DNA methyltransferase; EGFR = epidermal growth factor receptor; GBM = glioblastoma; HIF- $1 \alpha=$ hypoxia-inducible factor $1 \alpha$; IDH = isocitrate dehydrogenase; $\mathrm{MES}=$ mesenchymal; $\mathrm{NF}-\kappa \mathrm{B}=$ nuclear factor $\kappa \mathrm{B}$; PDGFR $\alpha=$ platelet-derived growth factor receptor $-\alpha$; PDK $=$ pyruvate dehydrogenase kinase; $\mathrm{PN}=$ proneural; $\mathrm{TCGA}=$ the Cancer Genome Atlas; TET $=$ ten-eleven translocation. in gliomas, the clinical and therapeutic/diagnostic implications, and the molecular subclasses of non-IDH1-mutated gliomas.

\section{History of IDH1 Mutations}

IDH1 mutations were initially reported in 2008; mutation of the arginine at codon 132 is the most frequent type, and these mutations are thought to lead to better overall survival..$^{51,77}$ The most common mutation is $\mathrm{R} 132 \mathrm{H}$ (arginine to histidine), which is observed in 80\%-90\% of IDH1 mutations in astrocytic and oligodendroglial gliomas. ${ }^{2,44,70,77}$ Other mutations in IDH1 are rare, decreasing in frequency from R132C to R132G to R132S., ${ }^{2,77}$ In cases in which IDH1 is not mutated, less frequent mutations at IDH2 have been reported at codon 172, with R172K being most frequent. ${ }^{51,77}$ Interestingly, the R132C mutation in IDH1 is seen exclusively in patients with Li-Fraumeni syndrome. ${ }^{71}$ Studies have shown that IDH1 mutations are predominant in secondary GBM $(>75 \%-80 \%)$ and are rare in primary GBM (5\%). ${ }^{44,51,70,77}$ It has been found that IDH1 is a marker of secondary GBM and that those primary GBMs diagnosed with IDH1 mutations may have been secondary gliomas that rapidly progressed to GBM with no early low-grade clinical symptoms experienced by patients, as elegantly reviewed by Ohgaki et al. ${ }^{47}$

IDH1 mutations are frequent in diffuse astrocytoma 
(WHO Grade II) and anaplastic astrocytoma (WHO Grade III) (80\%) and progress to secondary GBM. In addition to astrocytic tumors, IDH1 mutations frequently occur in oligodendroglial tumors, including oligodendroglioma (WHO Grade II) and anaplastic oligodendroglioma (WHO Grade III), ${ }^{44,70}$ which strongly suggests that IDH1 mutations occur early in gliomagenesis and occur in a progenitor cell that can give rise to both cell types. IDH1 mutations have not been reported in other CNS tumors such as ependymomas, medulloblastomas, meningiomas, and pilocytic astrocytomas and are very rare in spinal gliomas. ${ }^{6,33,34,44,45,50,51,55,70,77,78}$ Furthermore, IDH1 mutations have not been reported in pediatric gliomas such as pediatric GBM and diffuse infiltrating pontine gliomas, suggesting that they are restricted to astrocytomas and oligodendrogliomas in young adults. ${ }^{61,65,75,78}$ Gliomas in children are predominantly driven by mutations in histone genes $H 3 F 3 A$ or $H I S T I H 3 B$, with mutations at $\mathrm{K} 27 \mathrm{M}$ or G34V or G34R. It is interesting to note that mutations in IDH1 alter the epigenetic profile of these tumors (see below), as do these histone mutations in pediatric GBM. The nature of these mutations, restricted to certain ages and tumor types, suggest that pediatric and young adult gliomas may have different cells of origin. $.1,65,75,78$

\section{Metabolism in Brain Tumors and the Biological Roles of IDH1 Mutations}

Metabolic alterations are highly recurrent in gliomas. This is exemplified by a switch in splice isoforms from the adult pyruvate kinase muscle 1 (PKM1) to the fetal PKM2, which is believed to promote aerobic glycolysis in several cancers by altering the rate of glycolysis and diverting glycolytic intermediates into biosynthetic processes that are essential for tumor growth. ${ }^{13}$ Pyruvate dehydrogenase kinase 1 (PDK1) is another perpetrator of aerobic glycolysis that is upregulated in most cancer cells, including GBMs. PDK can inhibit pyruvate entry into the tricarboxylic acid (TCA) cycle by phosphorylating and inactivating the enzyme pyruvate dehydrogenase $(\mathrm{PDH}){ }^{43}$ Inhibitors of PDK1 such as dichloroacetate (DCA) have been shown to have anticancer effects by shifting metabolism from glycolysis to glucose oxidation and inducing apoptosis in many cancers, including GBMs. ${ }^{7,42}$ Hexokinase II (HKII) has also been reported to be altered and plays an important role in glioma tumor metabolism. ${ }^{72-74}$ Perhaps the most studied metabolic alterations in gliomas, including GBMs, are mutations in IDH1 and IDH2. Mutations in IDH1 lead to neomorphic activity of IDH1, producing a different metabolite, 2-hydroxyglutarate $(2 \mathrm{HG})$, that promotes a hypermethylator phenotype in addition to other phenotypes in gliomas and secondary GBMs ${ }^{14,46}$ (Fig. 1). IDH1 is an enzyme that participates in the citric acid (Krebs) cycle, converting isocitrate to $\alpha$-ketoglutarate ( $\alpha-\mathrm{KG}$ ), and is critical for generating adenosine triphosphate (ATP) for cellular energy ${ }^{21}{ }^{2} \mathrm{IDH} 1 / 2$ mutations are exclusively heterozygous, and it has been shown that the mutant version of IDH1 interacts with and reduces the activity of the wild-type enzyme. ${ }^{30,31}$ IDH1 mutations are considered gain-of-function mutations, resulting in the production of the oncometabolite $2 \mathrm{HG}$ from $\alpha-\mathrm{KG}^{14,54}$ IDH1 or IDH2 mutant gliomas contain higher levels of $2 \mathrm{HG}$ than do IDH wildtype tumors. ${ }^{14,31}$ In gliomas, the frequency of IDH1 mutations in codon 132 increases in the order R132L, R132S, R132G, R132C, to R132H, with R132H constituting more than $90 \%$ of all IDH1 mutations. ${ }^{2,77}$ It is surprising that 1 study demonstrated that the rare IDH1 R132 mutations produced more $2 \mathrm{HG}$ than did IDH1 $\mathrm{R} 132 \mathrm{H}$, and these findings may suggest natural selection against the rarer IDH1 R132 mutation in human glioma as a result of toxicity caused by high levels of D-2HG. ${ }^{52}$

IDH1 mutations and 2HG production have been shown to inhibit prolyl-hydroxylase (PHD) enzymes, which inhibit hypoxia-inducible factor $1 \alpha$ (HIF-1 $\alpha$ ), a major proangiogenic and proglycolysis transcription factor. ${ }^{76,79}$ IDH1 mutants in glioma and GBM cells have been shown to have elevated levels of HIF-1 $\alpha$ and HIF-1 $\alpha$ target genes, including GLUT1, VEGF, and PDK1. ${ }^{79}$ However, 1 study indicated that mutant IDH1 destabilized HIF-1 $\alpha$ in vitro, but the exact mechanism of how $2 \mathrm{HG}$ stabilizes HIF-1 $\alpha$ is not fully understood ${ }^{37}$ Recently, a transgenic mouse was generated using a conditional knock-in of IDH1 (R132H) in which activation was restricted to Nestin-expressing cells. ${ }^{59,60}$ In this study, the authors demonstrated that these mice expressed $2 \mathrm{HG}$ and elevated HIF-1 $\alpha$ levels and its gene transcriptional activity. Furthermore, these mice had defects in collagen and protein maturation, leading to a basement-membrane aberration and perinatal lethality. ${ }^{59}$ Whether IDH1 mutation is sufficient by itself to initiate glioma formation is still unknown.

\section{Biological Roles of IDH1 Mutations: IDH1-Driven Hypermethylator Phenotype}

A second alteration associated with IDH1 mutations is the presence of a $\mathrm{CpG}$ island hypermethylator phenotype (CIMP). From a genome-wide methylation profile analysis of astrocytomas and GBMs, it was reported that IDH1 mutations had unique $\mathrm{CpG}$ island methylation at a larger number of loci than did non-IDH1 mutant and primary GBMs. ${ }^{19,46}$ Several studies have shown that the introduction of mutant IDH1 into human astrocytes causes functional alterations of specific histone markers by impairing histone demethylation and the induction of DNA hypermethylation. Therefore, the IDH1 mutation is sufficient to establish a hypermethylation phenotype in gliomas. ${ }^{41,66}$ Furthermore, a single copy of the IDH1 R132H mutant was introduced into a human colon cancer cell line (HCT116) so that it was under the control of its endogenous promoter. ${ }^{17}$ This resulted in DNA methylation in more than $27,000 \mathrm{CpG}$ dinucleotides. ${ }^{17}$ Collectively, these data provide insight into the epigenetic alterations induced by IDH1 mutations and support a causal role for IDH1 (R132H/wild type) mutants in driving epigenetic instability in human cancer cells. One study demonstrated mechanistically that $2 \mathrm{HG}$ is a competitive inhibitor of multiple $\alpha$-KG-dependent dioxygenases, including histone demethylases and the ten-eleven translocation (TET) 


\section{IDH status and GBM}

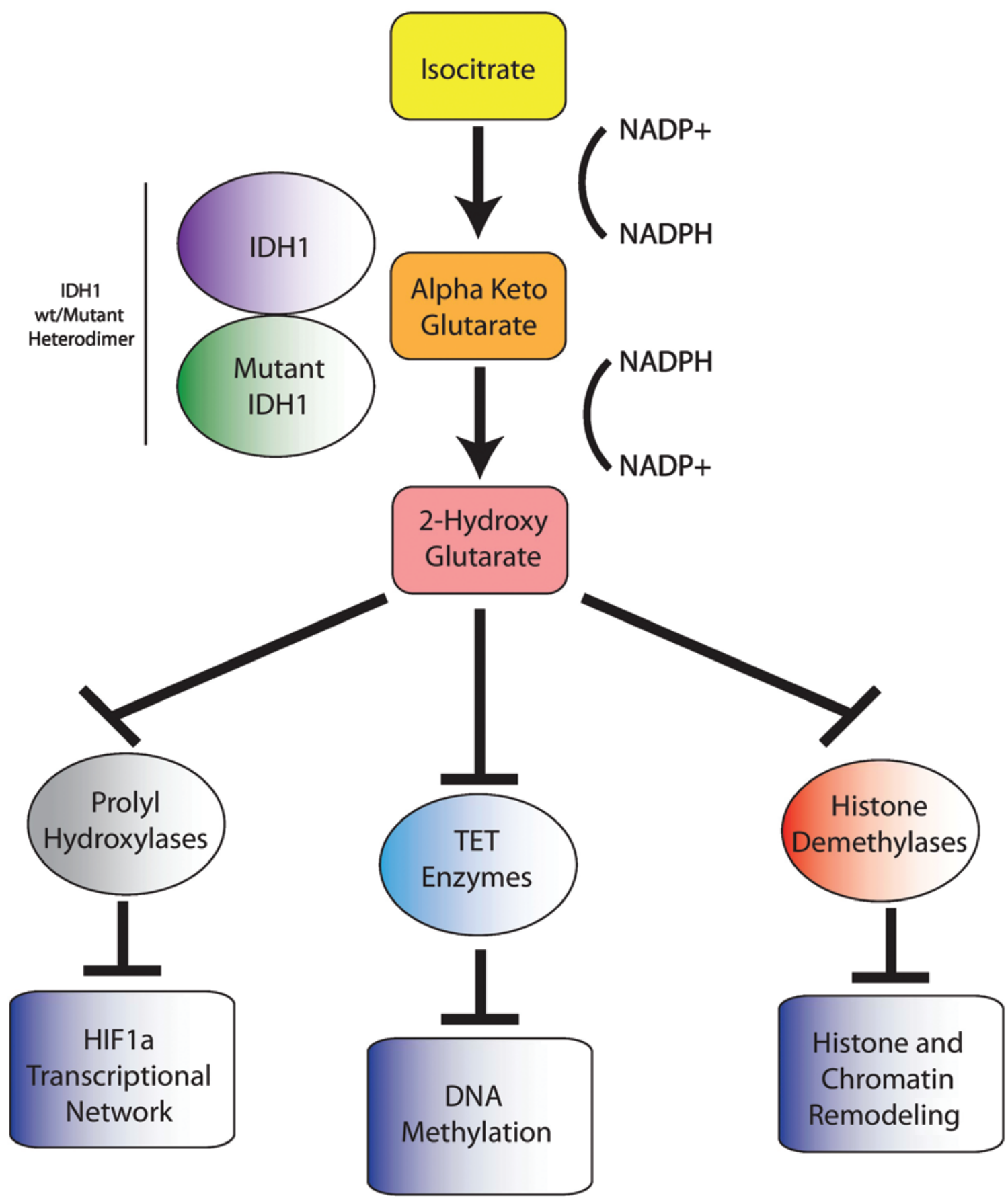

FIG. 1. Functions of IDH1 mutations. Wild-type (wt) and mutant IDH1 form a heterodimer. Isocitrate is converted into $\alpha$-KG by wild-type IDH1 and generates nicotinamide adenine dinucleotide phosphate (NADPH), whereas mutant IDH1 converts $\alpha-K G$ into $2 \mathrm{HG}$ and consumes the reducing agent NADPH in the reaction. $2 \mathrm{HG}$ has been shown to inhibit prolyl-hydroxylases, TET enzymes, and histone methylases, leading to the activation of HIF-1 $\alpha$ and aberrant epigenetic modifications in tumor cells.

family of 5-methylcytosine $(5 \mathrm{mC})$ hydroxylases. ${ }^{19,66,76}$ $2 \mathrm{HG}$ occupied the same space as $\alpha-\mathrm{KG}$ does in the active site of histone demethylases, and the expression of IDH1 and IDH2 mutants inhibited histone demethylation and $5 \mathrm{mC}$ hydroxylation ${ }^{19,66,76}$ (Fig. 1). In addition, in a study of a cohort of patients with acute myeloid leukemia (AML), IDH1/2 mutations were mutually exclusive, with mutations in the $\alpha-\mathrm{KG}-$ dependent enzyme TET2, and TET2 loss-of-function mutations are associated with epigenetic defects similar to those of IDH1/2 mutants. Consistent with these observations, the expression of IDH1/2 mutants impaired TET2 catalytic function in cancer cells and either expression of mutant IDH1/2 or Tet 2 depletionimpaired hematopoietic differentiation, suggesting that IDH1 mutations and TET enzyme inactivation share proleukemogenic effects. ${ }^{19}$ 


\section{Diagnostic and Prognostic Implications of IDH Mutations and Common Coexpressed Alterations}

It was initially demonstrated that IDH1 mutation was a positive prognostic marker, because patients with this mutation had significantly better overall survival. ${ }^{51}$ Several studies also showed that patients with GBMs or diffuse gliomas with IDH1 mutation have better overall survival and progression-free survival. ${ }^{16,23,25,26,28,57,67}$ Furthermore, mutant IDH1/2 associated with 1p/19q deletion prognosticates good outcomes for those with Grade III oligodendroglial tumors. ${ }^{28,57,67}$

Clinically diagnosed secondary GBM results in a median survival time of approximately 8 months, which is significantly longer than that for patients with primary GBM (approximately 5 months). ${ }^{48}$ Given the evidence that IDH1 mutations are restricted mostly to secondary GBMs and lower-grade gliomas, the IDH1 mutation as a prognostic marker of GBM may just reflect the 2 disease types. Although patients with the IDH1 mutation are generally younger, multivariate analysis has demonstrated that a patient's IDHI mutations are an independent prognostic factor even after adjusting for age, tumor grade, and $M G M T$ status. ${ }^{58}$ Two studies have demonstrated that patients with IDH1 mutations versus those with wild-type IDH1 had significantly better overall survival times when treated with surgery and radiotherapy (27 vs 11 months and 31 vs 16 months, respectively). ${ }^{44,77}$ In another study of patients with dedifferentiated low-grade astrocytomas that progressed after radiotherapy, the responses to temozolomide did not differ between IDHI mutant and wildtype tumors. ${ }^{16}$

IDH mutations are thought to arise early in gliomagenesis and persist during progression to secondary GBM. Therapies for recurrent gliomas often fail, which may be partly because the genomic alterations that drive the growth of recurrences are distinct from those in the initial tumor. One group studied this recurrence by sequencing the exomes of 23 initial low-grade gliomas and matched recurrent tumors from the same patients. ${ }^{32}$ This study found that in $43 \%$ of the cases, at least half of the mutations in the initial tumor were undetected at recurrence, including driver mutations in TP53, ATRX, SMARCA4, and BRAF. ${ }^{32}$ Of great interest is that the IDH1 status never changed between primary and recurrent gliomas, which suggests that these tumors are initiated by the clonal expansion of cells with IDH1 mutant function.

Approximately $65 \%$ of diffuse astrocytomas carry mutations in TP53, whereas oligodendrogliomas have frequent $1 \mathrm{p} / 19 \mathrm{q}$ loss..$^{39,49,53}$ IDH1/2 mutations are likely to occur before TP53 mutation or 1 p/19q loss, because low-grade diffuse gliomas that have only an IDH1 mutation are more frequent than those that carry only a TP53 mutation or harbor 1p/19q loss..$^{35,44,70}$ Therefore, an IDH1 mutation may be the initial alteration in a common cell of origin, with loss of $1 \mathrm{p} / 19 \mathrm{q}$ driving the tumor toward an oligodendroglial lineage. ${ }^{44,47,69,70}$ Recently, a genome-wide sequencing project demonstrated that mutations in the CIC gene (homolog of the Drosophila gene capicua) at $19 \mathrm{q} 13$ and in the FUBP1 gene at 1p are frequent in oligo- dendrogliomas but are rare or absent in diffuse astrocytomas, supporting the notion that that $1 p / 19 q$ with IDH1 mutation is essential for oligodendroglioma formation. ${ }^{3,29}$ Conversely, IDH1 mutations, followed by mutations in TP53 and ATRX (thalassemia/mental retardation syndrome X-linked) and its binding partner DAXX (deathassociated protein) may lead to diffuse astrocytoma formation. ATRX and DAXX are involved in incorporating histones into chromosomal sites of heterochromatin, notably telomeres. ${ }^{22,38}$ Therefore, dysfunction of the ATRX/ DAXX complex results in both widespread genomic instability and a phenomenon known as alternative lengthening of telomeres (ALT), a telomerase-independent mechanism of telomere maintenance that, analogous to telomerase activation, likely promotes cellular immortalization. $^{38,40}$

\section{Treatment and Clinical Testing Implications of IDH1 Mutations in Gliomas}

IDH1 mutation may lend itself as a diagnostic, predictive, or theranostic marker for treatments. To date, many clinical and molecular diagnostic laboratories sequence human tumors for IDH1 or IDH2 mutations. Furthermore, the generation of a monoclonal antibody against the most common IDH1 mutation $(\mathrm{R} 132 \mathrm{H})$ allows for immunohistochemical analysis of low-grade gliomas and GBMs. ${ }^{9,10}$ It is now possible, using MR spectrometry, to detect the presence of IDH mutant oncometabolite $2 \mathrm{HG},{ }^{1,12,18}$ which may allow for noninvasive detection of the grade or subtype of glioma and may be used as a method of evaluating responses to potential treatments against IDH mutant tumors.

The pharmaceutical company Agios has developed an IDH1 inhibitor (AGI-5198) that was shown in 2 separate studies to bind to and inhibit mutant IDH1 in a dosedependent manner. ${ }^{15,56}$ Under conditions of $2 \mathrm{HG}$ inhibition, the inhibitor induced the demethylation of histone H3K9me3 and the expression of genes associated with gliogenic differentiation with appreciable changes in genome-wide DNA methylation. ${ }^{15}$ Therefore, it seems promising that these inhibitors may enter Phase I clinical trials of patients with glioma harboring IDH1 mutations. In addition to directly targeting IDH1, there is growing evidence that disrupting protein function upstream or downstream of mutant IDH may yield promising novel therapeutic strategies. The targeting of glutaminase and glutamate carboxypeptidase II, an enzyme upstream of the mutated IDH1 pathway that produces glutamate from glutamine, yielded a strong antitumor response. ${ }^{62}$ IDHI mutation is also a driver of a hypermethylator phenotype, and therefore, DNA methyltransferase (DNMT) inhibitors and histone deacetylase inhibitors may also have therapeutic efficacy. One study demonstrated that efficient induction of differentiation and growth inhibition was observed in IDH1 mutant glioma cells by targeting the DNMT with the DNMT inhibitor decitabine. ${ }^{15}$ It should be noted that many of these targeted enzymes are expressed in normal cells, and targeting these proteins in normal tissue may cause nonspecific toxicity. The 
identification of IDH1 mutant synthetic lethal interactions using a genome-wide RNA interference screen or drug screen may reveal novel interactions with or regulators of mutant but not wild-type IDH1.

\section{Non-IDH1-Mutated Glioma Molecular Classes}

As mentioned earlier, IDH1 mutations are highly recurrent in low-grade gliomas and secondary GBMs (> $80 \%$ ). However, the majority of GBMs are not secondary; what is the molecular profile of these tumors? Our latest insight into the molecular pathogenesis of GBMs comes from recent large-scale multi-institutional studies and collaborative projects investigating the molecular signature of GBMs. Integrated genomic/transcriptome and epigenomic analysis resulted in a gene expressionbased molecular classification of GBMs into classical, mesenchymal (MES), proneural (PN), and neural subtypes, characterized by aberrations and gene expression of the epidermal growth factor receptor (EGFR), neurofibromatosis Type 1, and platelet-derived growth factor receptor $\alpha$ (PDGFR $\alpha)$. The studies have demonstrated that responses to aggressive chemotherapy and radiotherapy differ according to subtype. ${ }^{8,68}$ Using unsupervised hierarchical clustering, Verhaak et al. ${ }^{68}$ identified 4 molecular subtypes of GBM using an 840-gene signature that was validated in separate data sets. Each subtype was enriched for different mutation, genome, and transcript alterations. The PN subgroup was enriched for mutations in IDH1/2, mutation in TP53, and amplifications of PDGFR $\alpha$, cyclin-dependent kinase 6 (CDK6), CDK4, and MET. In addition, this group contained the highest percentage of young patients, likely because of the enrichment of IDH1 mutations, which is associated with younger patient age. The classical subtype is characterized by EGFR amplification and a loss of phosphatase and tensin homolog (PTEN). The classical subtype also harbors the mutant EGFR variant III (EGFRvIII) mutation, which is constitutively active and has an in-frame deletion of exons 2-7. The MES subclass is associated with poor overall survival, contains neurofibromatosis Type 1 mutations, and has a loss of TP53 and CDK inhibitor N2A (CDKN2A). Last, the neural subtype has elevated levels of neural markers such as NEFL but has no unique distinguishing alterations from other classes, although elevated rates of ERBB2 mutation were observed. Diffuse astrocytomas, oligodendrogliomas, and oligoastrocytomas all harbor a PN gene signature (62) similar to that of IDH1 mutations in GBMs, strongly suggesting that these tumors may arise from a common neural progenitor population. The molecular subtypes of gliomas are summarized in Fig. 2. Furthermore, each adult GBM subtype and pediatric GBM subtypes have unique methylation profiles in addition to their gene-expression profiles. ${ }^{8,65}$ This classification may support the notion that each subtype of GBM may arise from a unique cell of origin and that methylation profiling may be a key diagnostic tool for identifying subtypes of glioma or GBM with different genetic alterations.

\section{Subtype Switching in GBM}

Unlike the IDH mutant PN CIMP-positive subtype, it has been observed that multiple samples from tumors within the same patient can harbor all of the molecular subtypes of GBM. ${ }^{63}$ These results reveal the genomewide architecture of intratumor variability and heterogeneity that has not been accounted for in previous studies of GBM samples and warrant additional investigation. Several functional studies have also demonstrated that the molecular subtypes of GBM can undergo subtype switching in vitro and in vivo. One elegant study showed that patient-derived glioma sphere cultures that resemble either the PN or MES transcriptomal subtypes differ significantly in their biological characteristics. ${ }^{4}$ It is surprising that the authors found that a subset of the PN glioma sphere cultures underwent transition to a MES state in a tumor necrosis factor $-\alpha /$ nuclear factor $\kappa \mathrm{B}(\mathrm{NF}-\kappa \mathrm{B})-\mathrm{de}-$ pendent manner. They were then able to demonstrate that the MES signature, CD44 expression, and NF- $\kappa \mathrm{B}$ activation correlate with poor radiation response and shorter survival time in patients with $\mathrm{GBM} .{ }^{4}$ A second study identified the transcriptional coactivator with a PDZbinding motif (TAZ) to be highly associated with the MES network..$^{5}$ TAZ expression was lower in PN GBMs and lower-grade gliomas, which correlated with $\mathrm{CpG}$ island hypermethylation of the TAZ promoter compared with that of MES GBMs. Silencing of TAZ in MES glioma stem cells decreased the expression of MES markers, invasion, self-renewal, and tumor formation. ${ }^{5}$ Conversely, the overexpression of TAZ in PN glioma stem cells and murine neural stem cells induced MES marker expression. Another study recently demonstrated that radiation induced a marked shift away from a PN expression pattern toward a MES pattern in a transgenic mouse model. ${ }^{24}$ Mechanistically, radiation activated STAT3 and CEBPB, which have been suggested to be master regulators of a MES shift. ${ }^{24}$ In summary, there is evidence to support that subtype switching can occur through transcriptional reprogramming or even by treatment.

\section{IDH1 Mutations in Primary Versus Secondary GBMs}

As mentioned throughout this review, IDH1 mutations are seen almost exclusively in low-grade gliomas and secondary GBMs. Primary GBMs have been reported to have very low mutation rates in IDH1 or IDH2 (< 3\%-5\%). ${ }^{8,47,68}$ Conversely, a small percentage of Grade II diffuse gliomas lack alterations in either IDH or TP53 or $1 p / 19 q$ loss. Therefore, are these early-detected primary GBM lesions?

Low-grade gliomas and secondary GBMs lacking IDH1 mutations have infrequent TP53 mutations, and the patients with them have a shorter clinical history. ${ }^{44}$ One study demonstrated that secondary GBMs lacking IDH1 mutations had developed through progression from an anaplastic glioma (WHO Grade III), whereas the majority of secondary GBMs with IDH1 mutations had progressed from a WHO Grade II glioma. ${ }^{44}$ These results suggests the possibility that some tumors diagnosed as anaplastic as- 
S. Agnihotri, K. D. Aldape, and G. Zadeh

\section{Pediatric Glioma}

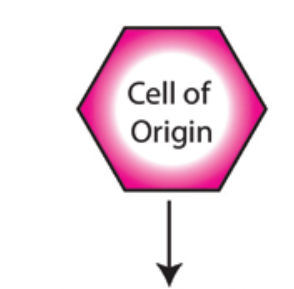

Histone Mutations

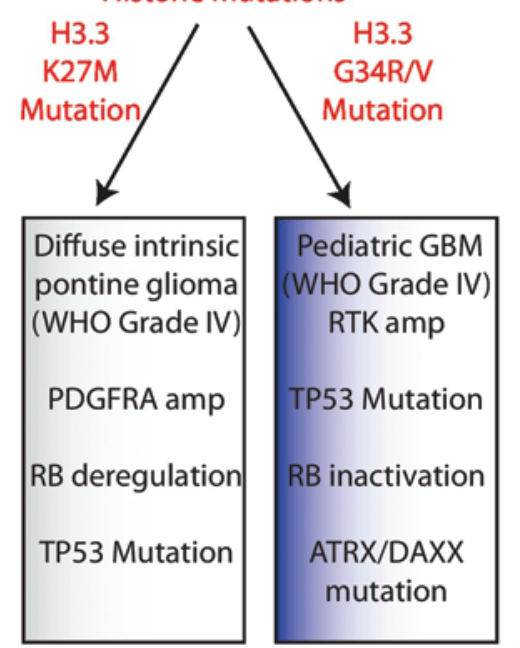

Glioma in Young Adults

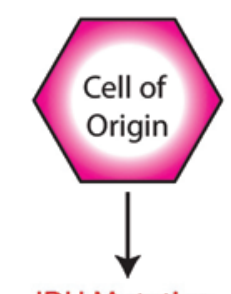

IDH Mutation
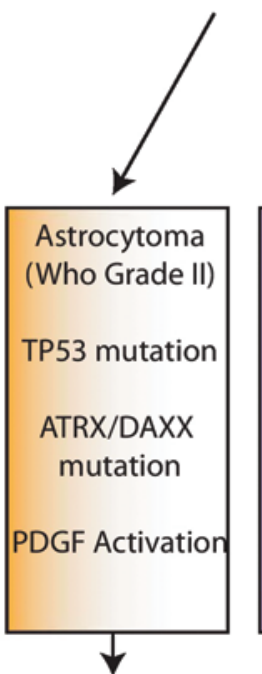
Anaplastic
Astrocytoma
(Who Grade III)
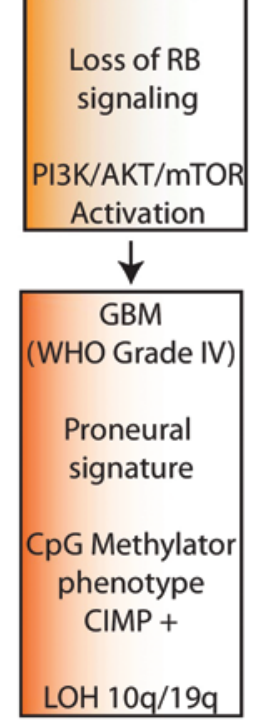
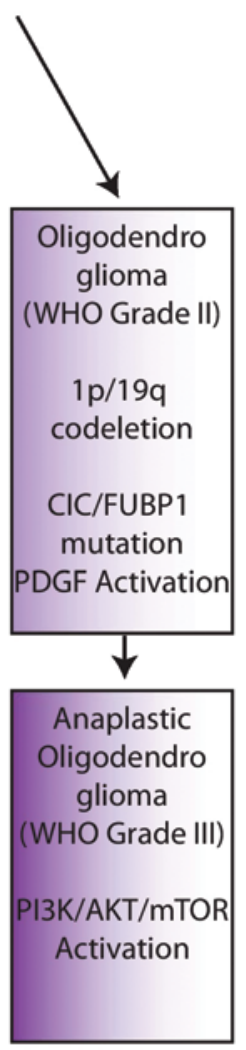

\section{Primary Glioblastoma (WHO Grade IV)} (Adults)

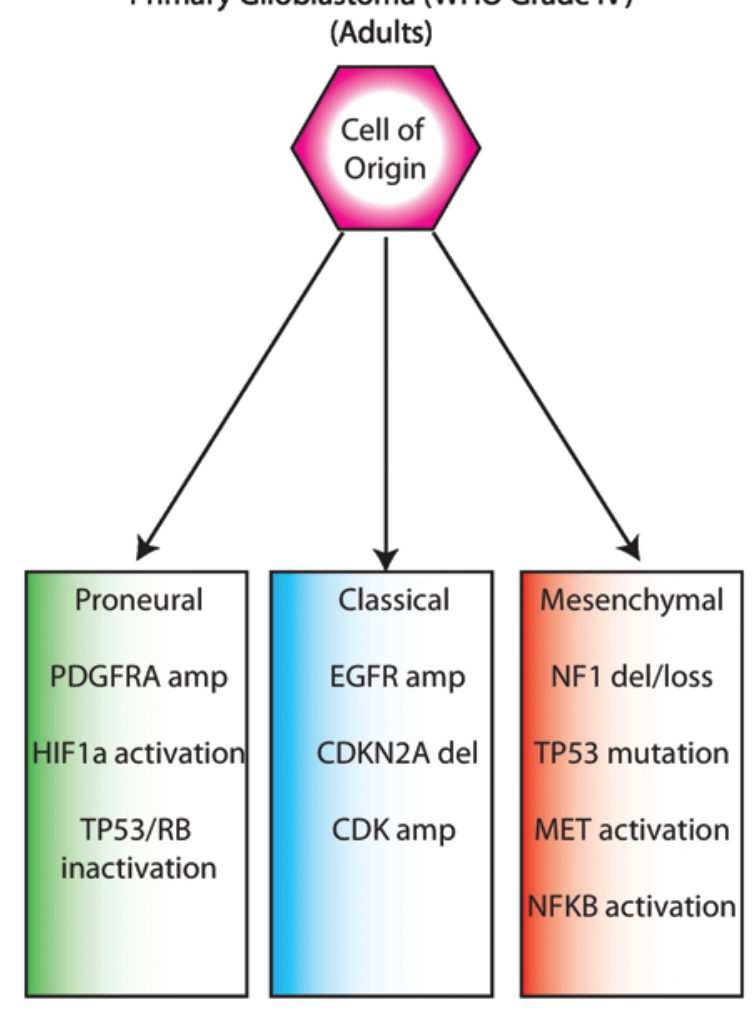

Fıg. 2. Transcriptional networks of gliomas. The classifications of pediatric and adult gliomas are based on molecular and genetic alterations. amp = amplification; del = deletion; MET = multiple receptor tyrosine; PDGFRA = PDGFR $\alpha$; RB = retinoblastoma; RTK = receptor tyrosine kinase.

trocytoma may in fact be primary GBMs that were misdiagnosed because of a lack of GBM diagnostic hallmarks, including necrosis or microvascular proliferation. To test this hypothesis, we explored the Cancer Genome Atlas (TCGA) data set of 262 low-grade gliomas and found that $75 \%$ of them harbored IDH1 mutations (Fig. 3A). ${ }^{8}$ Of great interest was that patients with IDH1 wild-type lowgrade gliomas showed enrichment of alterations in EGFR and loss of the CDKN2A locus, which are typically associated with primary GBMs. In support of this, EGFR and 

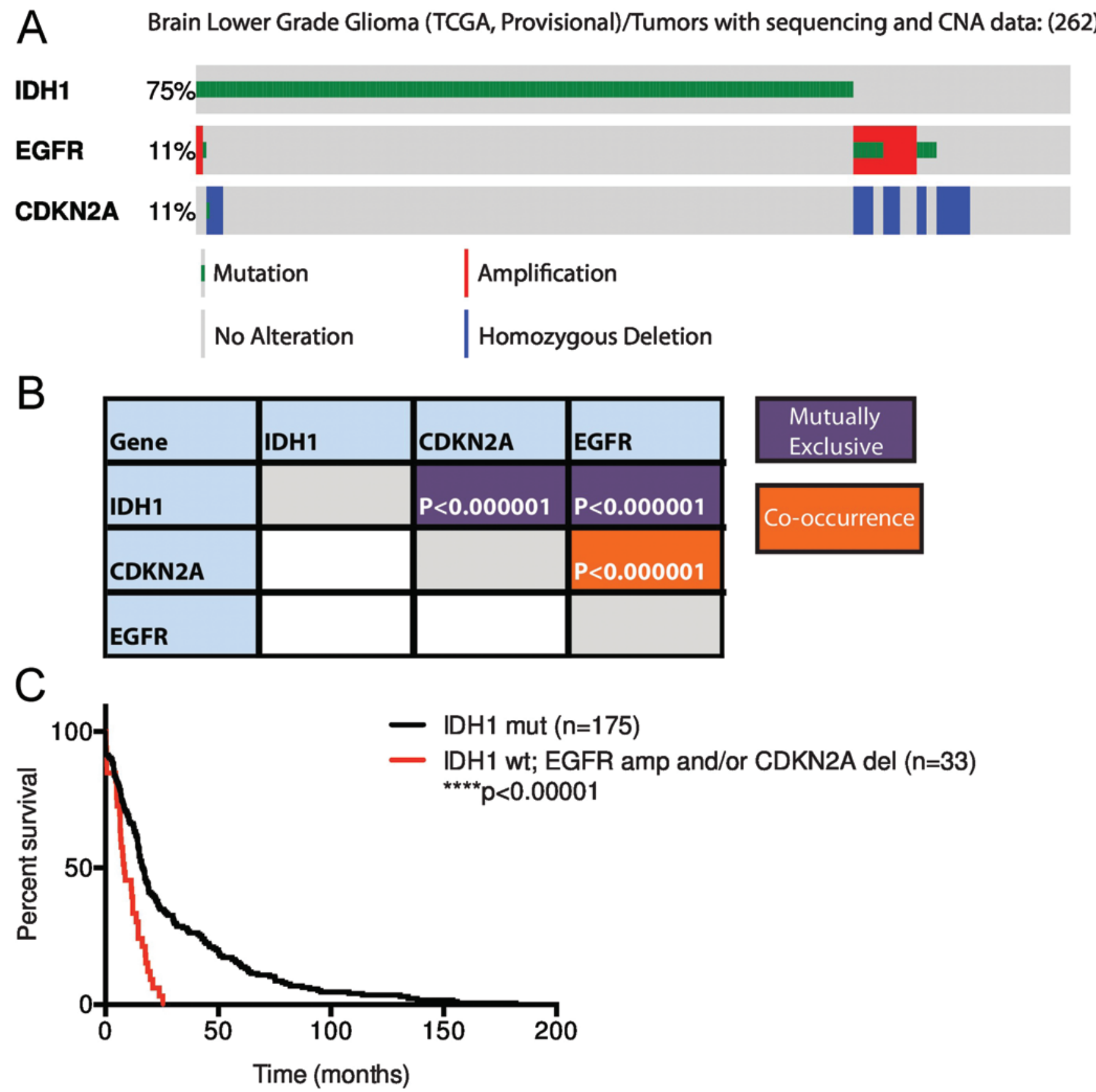

FIG. 3. TCGA analysis of low-grade gliomas. A: Onco-print analysis of 262 low-grade gliomas showing that IDH1 mutations are predominant. B: IDH1 mutations and EGFR amplifications or deletions in CDKN2A are mutually exclusive with the Fisher exact test. C: Survival analysis of IDH1 mutant gliomas versus IDH1 wild-type gliomas with an alteration in EGFR and/or CDKN2A, which are often seen in primary adult GBMs. Survival analysis was performed using a log-rank survival test. Provisional data were accessed through TCGA (http://cancergenome.nih.gov), and data were visualized and analyzed using the cBioPortal for Cancer Genomics (http://cbioportal.org). ${ }^{11,20}$

CDKN2A were mutually exclusive from IDH1 mutations (Fig. 3A and B). Again, in the low-grade glioma data set, patients with IDH1 mutations had better overall survival than patients with wild-type IDH1 harboring EGFR amplifications and/or CDKN2A homozygous deletions (Fig. 3C). The median survival time of patients with lowgrade gliomas with EGFR and CKN2A alterations was 8 months versus 16 months for those with low-grade glio- mas with mutant IDH1. This observation suggests that IDH1 wild-type non-CIMP-positive gliomas with EGFR amplification/mutation and loss of CDKN2A may in fact represent early-detected primary lesions.

\section{Conclusions}

In the last decade, we have benefited from an explo- 
sive wealth of knowledge identifying the molecular underpinnings of gliomas. We now know that the genetic landscape is diverse and carries unique alterations and different clinical histories. Emerging challenges will be the use of this wealth of data by biologists and clinicians to better understand gliomas and translate this knowledge into better prognostic, diagnostic, and treatment options for our patients.

\section{Disclosure}

The authors report no conflict of interest concerning the materials or methods used in this study or the findings specified in this paper.

Author contributions to the study and manuscript preparation include the following. Conception and design: all authors Acquisition of data: all authors. Analysis and interpretation of data: all authors. Drafting the article: Zadeh.

\section{References}

1. Andronesi OC, Rapalino O, Gerstner E, Chi A, Batchelor TT, Cahill DP, et al: Detection of oncogenic IDH1 mutations using magnetic resonance spectroscopy of 2-hydroxyglutarate. $\mathbf{J}$ Clin Invest 123:3659-3663, 2013

2. Balss J, Meyer J, Mueller W, Korshunov A, Hartmann C, von Deimling A: Analysis of the IDH1 codon 132 mutation in brain tumors. Acta Neuropathol 116:597-602, 2008

3. Bettegowda C, Agrawal N, Jiao Y, Sausen M, Wood LD, Hruban RH, et al: Mutations in CIC and FUBP1 contribute to human oligodendroglioma. Science 333:1453-1455, 2011

4. Bhat KP, Balasubramaniyan V, Vaillant B, Ezhilarasan R, Hummelink K, Hollingsworth F, et al: Mesenchymal differentiation mediated by $\mathrm{NF}-\kappa \mathrm{B}$ promotes radiation resistance in glioblastoma. Cancer Cell 24:331-346, 2013

5. Bhat KP, Salazar KL, Balasubramaniyan V, Wani K, Heathcock L, Hollingsworth F, et al: The transcriptional coactivator TAZ regulates mesenchymal differentiation in malignant glioma. Genes Dev 25:2594-2609, 2011

6. Bleeker FE, Lamba S, Leenstra S, Troost D, Hulsebos T, Vandertop WP, et al: IDH1 mutations at residue p.R132 (IDH1(R132)) occur frequently in high-grade gliomas but not in other solid tumors. Hum Mutat 30:7-11, 2009

7. Bonnet S, Archer SL, Allalunis-Turner J, Haromy A, Beaulieu $\mathrm{C}$, Thompson R, et al: A mitochondria-K+ channel axis is suppressed in cancer and its normalization promotes apoptosis and inhibits cancer growth. Cancer Cell 11:37-51, 2007

8. Brennan CW, Verhaak RG, McKenna A, Campos B, Noushmehr H, Salama SR, et al: The somatic genomic landscape of glioblastoma. Cell 155:462-477, 2013

9. Capper D, Reuss D, Schittenhelm J, Hartmann C, Bremer J, Sahm F, et al: Mutation-specific IDH1 antibody differentiates oligodendrogliomas and oligoastrocytomas from other brain tumors with oligodendroglioma-like morphology. Acta Neuropathol 121:241-252, 2011

10. Capper D, Weissert S, Balss J, Habel A, Meyer J, Jäger D, et al: Characterization of R132H mutation-specific IDH1 antibody binding in brain tumors. Brain Pathol 20:245-254, 2010

11. Cerami E, Gao J, Dogrusoz U, Gross BE, Sumer SO, Aksoy $\mathrm{BA}$, et al: The cBio cancer genomics portal: an open platform for exploring multidimensional cancer genomics data. Cancer Discov 2:401-404, 2012 (Erratum in Cancer Discov 2:960, 2012)

12. Choi C, Ganji SK, DeBerardinis RJ, Hatanpaa KJ, Rakheja D, Kovacs Z, et al: 2-hydroxyglutarate detection by magnetic resonance spectroscopy in IDH-mutated patients with gliomas. Nat Med 18:624-629, 2012

13. Christofk HR, Vander Heiden MG, Wu N, Asara JM, Cantley
LC: Pyruvate kinase M2 is a phosphotyrosine-binding protein. Nature 452:181-186, 2008

14. Dang L, White DW, Gross S, Bennett BD, Bittinger MA, Driggers EM, et al: Cancer-associated IDH1 mutations produce 2-hydroxyglutarate. Nature 462:739-744, 2009

15. Davis M, Pragani R, Popovici-Muller J, Gross S, Thorne N, Salituro F, et al: ML309: a potent inhibitor of R132H mutant IDH1 capable of reducing 2-hydroxyglutarate production in U87 MG glioblastoma cells. May 8, 2013. Probe Reports from the NIH Molecular Libraries Program. (http://www. ncbi.nlm.nih.gov/books/NBK153220/) [Accessed October 12, 2014]

16. Dubbink HJ, Taal W, van Marion R, Kros JM, van Heuvel I, Bromberg JE, et al: IDH1 mutations in low-grade astrocytomas predict survival but not response to temozolomide. Neurology 73:1792-1795, 2009

17. Duncan CG, Barwick BG, Jin G, Rago C, Kapoor-Vazirani P, Powell DR, et al: A heterozygous IDH1R132H/WT mutation induces genome-wide alterations in DNA methylation. Genome Res 22:2339-2355, 2012

18. Elkhaled A, Jalbert LE, Phillips JJ, Yoshihara HA, Parvataneni R, Srinivasan R, et al: Magnetic resonance of 2-hydroxyglutarate in IDH1-mutated low-grade gliomas. Sci Transl Med 4:116ra115, 2012

19. Figueroa ME, Abdel-Wahab O, Lu C, Ward PS, Patel J, Shih A, et al: Leukemic IDH1 and IDH2 mutations result in a hypermethylation phenotype, disrupt TET2 function, and impair hematopoietic differentiation. Cancer Cell 18:553-567, 2010

20. Gao J, Aksoy BA, Dogrusoz U, Dresdner G, Gross B, Sumer $\mathrm{SO}$, et al: Integrative analysis of complex cancer genomics and clinical profiles using the cBioPortal. Sci Signal 6:pl1, 2013

21. Geisbrecht BV, Gould SJ: The human PICD gene encodes a cytoplasmic and peroxisomal NADP(+)-dependent isocitrate dehydrogenase. J Biol Chem 274:30527-30533, 1999

22. Goldberg AD, Banaszynski LA, Noh KM, Lewis PW, Elsaesser SJ, Stadler S, et al: Distinct factors control histone variant H3.3 localization at specific genomic regions. Cell 140:678-691, 2010

23. Gorovets D, Kannan K, Shen R, Kastenhuber ER, Islamdoust $\mathrm{N}$, Campos $\mathrm{C}$, et al: IDH mutation and neuroglial developmental features define clinically distinct subclasses of lower grade diffuse astrocytic glioma. Clin Cancer Res 18:24902501,2012

24. Halliday J, Helmy K, Pattwell SS, Pitter KL, LaPlant Q, Ozawa T, et al: In vivo radiation response of proneural glioma characterized by protective p53 transcriptional program and proneural-mesenchymal shift. Proc Natl Acad Sci U S A 111:5248-5253, 2014

25. Hartmann C, Hentschel B, Wick W, Capper D, Felsberg J, Simon M, et al: Patients with IDH1 wild type anaplastic astrocytomas exhibit worse prognosis than IDH1-mutated glioblastomas, and IDH1 mutation status accounts for the unfavorable prognostic effect of higher age: implications for classification of gliomas. Acta Neuropathol 120:707-718, 2010

26. Hartmann C, Meyer J, Balss J, Capper D, Mueller W, Christians A, et al: Type and frequency of IDH1 and IDH2 mutations are related to astrocytic and oligodendroglial differentiation and age: a study of 1,010 diffuse gliomas. Acta Neuropathol 118:469-474, 2009

27. Hegi ME, Diserens AC, Godard S, Dietrich PY, Regli L, Ostermann S, et al: Clinical trial substantiates the predictive value of O-6-methylguanine-DNA methyltransferase promoter methylation in glioblastoma patients treated with temozolomide. Clin Cancer Res 10:1871-1874, 2004

28. Houillier C, Wang X, Kaloshi G, Mokhtari K, Guillevin R, Laffaire J, et al: IDH1 or IDH2 mutations predict longer survival and response to temozolomide in low-grade gliomas. Neurology 75:1560-1566, 2010

29. Jiao Y, Killela PJ, Reitman ZJ, Rasheed AB, Heaphy CM, 
de Wilde RF, et al: Frequent ATRX, CIC, FUBP1 and IDH1 mutations refine the classification of malignant gliomas. Oncotarget 3:709-722, 2012

30. Jin G, Reitman ZJ, Duncan CG, Spasojevic I, Gooden DM, Rasheed BA, et al: Disruption of wild-type IDH1 suppresses D-2-hydroxyglutarate production in IDH1-mutated gliomas. Cancer Res 73:496-501, 2013

31. Jin G, Reitman ZJ, Spasojevic I, Batinic-Haberle I, Yang J, Schmidt-Kittler O, et al: 2-hydroxyglutarate production, but not dominant negative function, is conferred by glioma-derived NADP-dependent isocitrate dehydrogenase mutations. PLoS ONE 6:e16812, 2011

32. Johnson BE, Mazor T, Hong C, Barnes M, Aihara K, McLean $\mathrm{CY}$, et al: Mutational analysis reveals the origin and therapydriven evolution of recurrent glioma. Science 343:189-193, 2014

33. Jones DT, Hutter B, Jäger N, Korshunov A, Kool M, Warnatz HJ, et al: Recurrent somatic alterations of FGFR1 and NTRK2 in pilocytic astrocytoma. Nat Genet 45:927-932, 2013

34. Jones DT, Jäger N, Kool M, Zichner T, Hutter B, Sultan M, et al: Dissecting the genomic complexity underlying medulloblastoma. Nature 488:100-105, 2012

35. Kim YH, Nobusawa S, Mittelbronn M, Paulus W, Brokinkel B, Keyvani K, et al: Molecular classification of low-grade diffuse gliomas. Am J Pathol 177:2708-2714, 2010

36. Kleihues P, Cavanee WK (eds): World Health Organization Classification of Tumors. Pathology and Genetics: Tumours of the Nervous System. Lyon: IARC Press, 2000

37. Koivunen P, Lee S, Duncan CG, Lopez G, Lu G, Ramkissoon $\mathrm{S}$, et al: Transformation by the (R)-enantiomer of 2-hydroxyglutarate linked to EGLN activation. Nature 483:484-488, 2012

38. Lewis PW, Elsaesser SJ, Noh KM, Stadler SC, Allis CD: Daxx is an H3.3-specific histone chaperone and cooperates with ATRX in replication-independent chromatin assembly at telomeres. Proc Natl Acad Sci U S A 107:14075-14080, 2010

39. Louis DN, Ohgaki H, Wiestler OD, Cavenee WK, Burger PC, Jouvet A, et al: The 2007 WHO classification of tumours of the central nervous system. Acta Neuropathol 114:97-109, 2007

40. Lovejoy CA, Li W, Reisenweber S, Thongthip S, Bruno J, de Lange T, et al: Loss of ATRX, genome instability, and an altered DNA damage response are hallmarks of the alternative lengthening of telomeres pathway. PLoS Genet 8:e1002772, 2012

41. Lu C, Ward PS, Kapoor GS, Rohle D, Turcan S, Abdel-Wahab $\mathrm{O}$, et al: IDH mutation impairs histone demethylation and results in a block to cell differentiation. Nature 483:474-478, 2012

42. Michelakis ED, Sutendra G, Dromparis P, Webster L, Haromy A, Niven E, et al: Metabolic modulation of glioblastoma with dichloroacetate. Sci Transl Med 2:31ra34, 2010

43. Michelakis ED, Webster L, Mackey JR: Dichloroacetate (DCA) as a potential metabolic-targeting therapy for cancer. Br J Cancer 99:989-994, 2008

44. Nobusawa S, Watanabe T, Kleihues P, Ohgaki H: IDH1 mutations as molecular signature and predictive factor of secondary glioblastomas. Clin Cancer Res 15:6002-6007, 2009

45. Northcott PA, Shih DJ, Peacock J, Garzia L, Morrissy AS, Zichner T, et al: Subgroup-specific structural variation across 1,000 medulloblastoma genomes. Nature 488:49-56, 2012

46. Noushmehr H, Weisenberger DJ, Diefes K, Phillips HS, Pujara $\mathrm{K}$, Berman $\mathrm{BP}$, et al: Identification of a $\mathrm{CpG}$ island methylator phenotype that defines a distinct subgroup of glioma. Cancer Cell 17:510-522, 2010

47. Ohgaki H, Burger P, Kleihues P: Definition of primary and secondary glioblastoma-response. Clin Cancer Res 20:2013, 2014 (Letter)

48. Ohgaki H, Kleihues P: Genetic pathways to primary and secondary glioblastoma. Am J Pathol 170:1445-1453, 2007
49. Okamoto Y, Di Patre PL, Burkhard C, Horstmann S, Jourde B, Fahey M, et al: Population-based study on incidence, survival rates, and genetic alterations of low-grade diffuse astrocytomas and oligodendrogliomas. Acta Neuropathol 108:49-56, 2004

50. Parker M, Mohankumar KM, Punchihewa C, Weinlich R, Dalton JD, Li Y, et al: C11orf95-RELA fusions drive oncogenic NF- $\mathrm{KB}$ signalling in ependymoma. Nature 506:451455, 2014 (Erratum in Nature 508:554, 2014)

51. Parsons DW, Jones S, Zhang X, Lin JC, Leary RJ, Angenendt $\mathrm{P}$, et al: An integrated genomic analysis of human glioblastoma multiforme. Science 321:1807-1812, 2008

52. Pusch S, Schweizer L, Beck AC, Lehmler JM, Weissert S, Balss J, et al: D-2-Hydroxyglutarate producing neo-enzymatic activity inversely correlates with frequency of the type of isocitrate dehydrogenase 1 mutations found in glioma. Acta Neuropathol Commun 2:19, 2014

53. Reifenberger G, Louis DN: Oligodendroglioma: toward molecular definitions in diagnostic neuro-oncology. J Neuropathol Exp Neurol 62:111-126, 2003

54. Reitman ZJ, Jin G, Karoly ED, Spasojevic I, Yang J, Kinzler $\mathrm{KW}$, et al: Profiling the effects of isocitrate dehydrogenase 1 and 2 mutations on the cellular metabolome. Proc Natl Acad Sci U S A 108:3270-3275, 2011

55. Robinson G, Parker M, Kranenburg TA, Lu C, Chen X, Ding L, et al: Novel mutations target distinct subgroups of medulloblastoma. Nature 488:43-48, 2012

56. Rohle D, Popovici-Muller J, Palaskas N, Turcan S, Grommes C, Campos C, et al: An inhibitor of mutant IDH1 delays growth and promotes differentiation of glioma cells. Science 340:626-630, 2013

57. Sabha N, Knobbe CB, Maganti M, Al Omar S, Bernstein M, Cairns R, et al: Analysis of IDH mutation, 1p/19q deletion, and PTEN loss delineates prognosis in clinical low-grade diffuse gliomas. Neuro Oncol 16:914-923, 2014

58. Sanson M, Marie Y, Paris S, Idbaih A, Laffaire J, Ducray F, et al: Isocitrate dehydrogenase 1 codon 132 mutation is an important prognostic biomarker in gliomas. J Clin Oncol 27:4150-4154, 2009

59. Sasaki M, Knobbe CB, Itsumi M, Elia AJ, Harris IS, Chio II, et al: D-2-hydroxyglutarate produced by mutant IDH1 perturbs collagen maturation and basement membrane function. Genes Dev 26:2038-2049, 2012

60. Sasaki M, Knobbe CB, Munger JC, Lind EF, Brenner D, Brüstle A, et al: IDH1(R132H) mutation increases murine haematopoietic progenitors and alters epigenetics. Nature 488:656-659, 2012

61. Schwartzentruber J, Korshunov A, Liu XY, Jones DT, Pfaff E, Jacob K, et al: Driver mutations in histone H3.3 and chromatin remodelling genes in paediatric glioblastoma. Nature 482:226-231, 2012

62. Seltzer MJ, Bennett BD, Joshi AD, Gao P, Thomas AG, Ferraris DV, et al: Inhibition of glutaminase preferentially slows growth of glioma cells with mutant IDH1. Cancer Res 70:8981-8987, 2010

63. Sottoriva A, Spiteri I, Piccirillo SG, Touloumis A, Collins VP, Marioni JC, et al: Intratumor heterogeneity in human glioblastoma reflects cancer evolutionary dynamics. Proc Natl Acad Sci U S A 110:4009-4014, 2013

64. Stupp R, Mason WP, van den Bent MJ, Weller M, Fisher B, Taphoorn MJ, et al: Radiotherapy plus concomitant and adjuvant temozolomide for glioblastoma. N Engl J Med 352:987996, 2005

65. Sturm D, Witt H, Hovestadt V, Khuong-Quang DA, Jones DT, Konermann C, et al: Hotspot mutations in H3F3A and IDH1 define distinct epigenetic and biological subgroups of glioblastoma. Cancer Cell 22:425-437, 2012

66. Turcan S, Rohle D, Goenka A, Walsh LA, Fang F, Yilmaz E, et al: IDH1 mutation is sufficient to establish the glioma hypermethylator phenotype. Nature 483:479-483, 2012 
67. van den Bent MJ, Dubbink HJ, Marie Y, Brandes AA, Taphoorn MJ, Wesseling P, et al: IDH1 and IDH2 mutations are prognostic but not predictive for outcome in anaplastic oligodendroglial tumors: a report of the European Organization for Research and Treatment of Cancer Brain Tumor Group. Clin Cancer Res 16:1597-1604, 2010

68. Verhaak RG, Hoadley KA, Purdom E, Wang V, Qi Y, Wilkerson MD, et al: Integrated genomic analysis identifies clinically relevant subtypes of glioblastoma characterized by abnormalities in PDGFRA, IDH1, EGFR, and NF1. Cancer Cell 17:98-110, 2010

69. Watanabe T, Nakamura M, Kros JM, Burkhard C, Yonekawa Y, Kleihues P, et al: Phenotype versus genotype correlation in oligodendrogliomas and low-grade diffuse astrocytomas. Acta Neuropathol 103:267-275, 2002

70. Watanabe T, Nobusawa S, Kleihues P, Ohgaki H: IDH1 mutations are early events in the development of astrocytomas and oligodendrogliomas. Am J Pathol 174:1149-1153, 2009

71. Watanabe T, Vital A, Nobusawa S, Kleihues P, Ohgaki H: Selective acquisition of IDH1 R132C mutations in astrocytomas associated with Li-Fraumeni syndrome. Acta Neuropathol 117:653-656, 2009

72. Wolf A, Agnihotri S, Guha A: Targeting metabolic remodeling in glioblastoma multiforme. Oncotarget 1:552-562, 2010

73. Wolf A, Agnihotri S, Micallef J, Mukherjee J, Sabha N, Cairns $\mathrm{R}$, et al: Hexokinase 2 is a key mediator of aerobic glycolysis and promotes tumor growth in human glioblastoma multiforme. J Exp Med 208:313-326, 2011

74. Wolf A, Agnihotri S, Munoz D, Guha A: Developmental profile and regulation of the glycolytic enzyme hexokinase 2 in normal brain and glioblastoma multiforme. Neurobiol Dis 44:84-91, 2011

75. Wu G, Broniscer A, McEachron TA, Lu C, Paugh BS, Becksfort J, et al: Somatic histone $\mathrm{H} 3$ alterations in pediatric diffuse intrinsic pontine gliomas and non-brainstem glioblastomas. Nat Genet 44:251-253, 2012

76. Xu W, Yang H, Liu Y, Yang Y, Wang P, Kim SH, et al: Oncometabolite 2-hydroxyglutarate is a competitive inhibitor of $\alpha$-ketoglutarate-dependent dioxygenases. Cancer Cell 19: 17-30, 2011

77. Yan H, Parsons DW, Jin G, McLendon R, Rasheed BA, Yuan W, et al: IDH1 and IDH2 mutations in gliomas. N Engl J Med 360:765-773, 2009

78. Zhang J, Wu G, Miller CP, Tatevossian RG, Dalton JD, Tang $\mathrm{B}$, et al: Whole-genome sequencing identifies genetic alterations in pediatric low-grade gliomas. Nat Genet 45:602-612, 2013

79. Zhao S, Lin Y, Xu W, Jiang W, Zha Z, Wang P, et al: Gliomaderived mutations in IDH1 dominantly inhibit IDH1 catalytic activity and induce HIF-1alpha. Science 324:261-265, 2009

Manuscript submitted August 15, 2014.

Accepted September 22, 2014.

Please include this information when citing this paper: DOI: 10.3171/2014.9.FOCUS14505.

Address correspondence to: Gelareh Zadeh, M.D., Ph.D., Arthur and Sonia Labatt Brain Tumour Research Centre, The Hospital for Sick Children, 4-439 West Wing, TWH, 399 Bathurst Ave., Toronto, ON M5T 2S8, Canada. email: gelareh.zadeh@uhn.ca. 